\title{
PPV-HB: Harmonic Balance for Oscillator/PLL Phase Macromodels
}

\author{
Ting Mei and Jaijeet Roychowdhury \\ \{meiting, jr\}@umn.edu \\ University of Minnesota, Twin Cities, USA
}

\begin{abstract}
A unique feature of oscillators is that small but sustained external perturbations lead to unboundedly large changes in phase, thereby making standard harmonic balance (HB) inapplicable to realistic oscillator phase macromodels. In this paper, we rectify this situation by presenting a novel extension of $\mathrm{HB}$ that is capable of handling oscillator phase macromodels. Key to the new method, termed PPV-HB, is a formulation that separates unboundedly increasing phase terms from the bounded, periodic components. PPV-HB can be used not only on individual oscillators, but it also enables the application of HB-like techniques for simulating systemlevel equation systems composed of higher-level macromodels of blocks. We validate PPV-HB on individual oscillators and a PLL system, demonstrating excellent matches with transient simulation using phase macromodels. Speedups of 1-2 orders of magnitude are obtained, over and above additional speedups of another 2-3 orders of magnitude that stem from using macromodels (as opposed to full circuit simulation).
\end{abstract}

\section{INTRODUCTION}

Oscillators - e.g., LC and ring oscillators, voltage-controlled oscillators (VCOs) and clocks - are widely used in electronic systems. They are often used as information carriers in communication systems, or as time references in clocked systems. For example, VCOs are key components of phase-locked loops (PLLs), which are widely used for clock generation and recovery, FM demodulation, frequency synthesis, etc. When perturbed by external signals, oscillators can change their natural frequency; a well-known effect, known as injection locking/pulling, involves the oscillator's moving its natural frequency towards that of a small external signal. Injection locking (IL) can be a nuisance in design as well as a useful phenomenon that is exploited in frequency dividers [1,2], IL-aided PLLs [3] and quadrature oscillator designs [4]. Therefore, analyzing oscillators' responses under perturbations is an important component of the oscillator design process.

The simulation of oscillators under perturbations presents unique challenges, however, since traditional SPICE-like simulation can be very inefficient and inaccurate. The fundamental reason for this is that oscillators feature neutral phase stability, which causes small numerical errors in phase to accumulate without limit during simulation. As a result, much smaller time-steps are required for oscillators than for other circuits during transient simulations.

To alleviate efficiency and accuracy problems during transient simulation of oscillators, a variety of specialized techniques have been proposed (e.g., [5-7]). A commonly used class of approaches relies on macromodelling the phase response of oscillators to external perturbations. A linear time-varying phase macromodel was first proposed by Kärtner [5,6] and later generalized to a time-shifted nonlinear one [7].

Permission to make digital or hard copies of all or part of this work for personal or classroom use is granted without fee provided that copies are not made or distributed for profit or commercial advantage and that copies bear this notice and the full citation on the first page. To copy otherwise, to republish, to post on servers or to redistribute to lists, requires prior specific permission and/or a fee.

ICCAD’06, November 5-9, 2006, San Jose, CA

Copyright 2006 ACM 1-59593-389-1/06/0011_..\$5.00
By reducing an oscillator system, normally described by differential algebraic equations (DAEs) of moderate or large size, to a system of size 1 (a scalar phase equation), these approaches improve simulation efficiency dramatically. However, the solution of the phase equation itself is usually performed via transient analysis [8]. Especially when oscillators are embedded in larger systems, such as PLLs, transient simulation, which can last thousands of cycles with each cycle requiring small time-steps, can take time.

Also, in practice, tasks such as predicting injection locking, performing oscillator natural frequency sweeps with respect to control, supply, etc., voltages, are frequently conducted during oscillator design. For these purposes, transient analysis on phase macromodels [8] is less than ideal. For example, it may take very many cycles of phase simulation to determine whether an oscillator is locked (or not locked) to an external signal. This is especially true for signals close to the lock range [9] of oscillators. From observing transient results of phase variations of an oscillator over an inadequate number of cycles, it is quite possible to draw wrong conclusions about the oscillator's state of lock (as we demonstrate later).

In this paper, we present an extension of Harmonic Balance (HB) [10-12], termed PPV-HB, suitable for application to nonlinear phase equations. Unlike normal DAE systems arising from circuits, where all unknowns (physical node voltages and branch currents) are periodic, the oscillator phase equation has unboundedly increasing terms. Therefore, standard HB cannot be applied directly to phase macromodels of oscillators. A key advance of our technique is the decomposition of phase variations into two parts: an unboundedly increasing part and a bounded periodic part. This leads to a modified phase equation formulation which is suitable for HB simulations.

By avoiding transient analysis of the phase equation, PPV-HB achieves further speedup in predicting oscillator behaviours. In addition, our technique can be extended to analyze oscillators under DC perturbation, which is useful for investigating oscillator frequency sweeps to power supply voltages. The main modification is the use of an extra unknown to capture the unboundedly increasing component of the phase. An extra equation is also added to help solve the system.

A key benefit of our technique is that it enables the use of $\mathrm{HB}$ for system-level simulations of PLLs using phase macromodels. The steady state of locked PLLs can be obtained by applying HB in the phase domain, with the help of our modified phase equation, resulting in increased simulation efficiency, accuracy and convenience over transient simulation of macromodels.

We demonstrate PPV-HB on LC and ring oscillators, as well as on a PLL. Simulation results show good agreement with transient simulation of both the original phase equation and the full systems. We obtain speedups of 1-2 orders of magnitude over transient simulation of phase macromodels. This translates to speedups of 3-4 orders of magnitude over full system transient simulations [8].

The remainder of the paper is organized as follows. In Section II, we review the nonlinear oscillator phase macromodel and the PLL phase domain macromodel based on it. In Section III, we present the PPV- 
$\mathrm{HB}$ technique for oscillator nonlinear phase macromodels. In Section IV, we apply PPV-HB to LC and ring oscillators, as well as PLLs, demonstrating large speedups over transient simulations.

\section{NONLINEAR PHASE MACROMODELS}

In this section, we first briefly review nonlinear phase macromodels [7] for oscillators. We then demonstrate their use in PLL phase domain simulations.

\section{A. Nonlinear phase macromodels for oscillators}

Oscillator circuits under perturbation can be described by the DAE system

$$
\frac{d q(x)}{d t}+f(x)=b(t),
$$

where $b(t)$ is a small perturbation signal and $x(t)$ is a vector of circuit unknowns (such as node voltages and branch currents). In [7], the solution to (1) is assumed to be:

$$
x(t)=x_{s}(t+\alpha(t))+y(t) .
$$

Here $x_{s}(t)$ is the steady state solution of the unperturbed oscillator, i.e., when $b(t)=0$ in (1). The effects of perturbations on oscillators are captured in two parts, with $\alpha(t)$ and $y(t)$ representing phase variations and amplitude variations, respectively. The phase variations $\alpha(t)$ can grow unboundedly with time while the amplitude variations can be shown to always remain small (if $b(t)$ is small).

The phase variation $\alpha(t)$ is obtained by solving a nonlinear scalar equation:

$$
\frac{d \alpha(t)}{d t}=v_{1}^{T}(t+\alpha(t)) \cdot b(t) .
$$

Here $v_{1}^{T}(t)$ is the perturbation projection vector (PPV), a function of time which has the same period that of the free-running oscillator and the same size as the circuit unknown's vector $x(t)$. The PPV represents "nonlinear phase sensitivities" of the oscillator to the perturbations injected at any nodes or branch. The PPV can be calculated numerically using two basic approaches, the monodromy matrix method [7] or the $A x=b$ technique in [13].

The phase equation (3), which is a size 1 system, can be used as a phase macromodel for the oscillator in larger systems such as PLLs. By simulating the smaller macromodel-based system, large speedups over simulation of the original full circuits can be obtained.

\section{B. PLL simulation using VCO phase macromodels}

The block diagram of a PLL is shown in Figure 1. It consists of 4 major components: a phase detector (PD), a low-pass filter (LPF), a VCO and a frequency divider (FD).

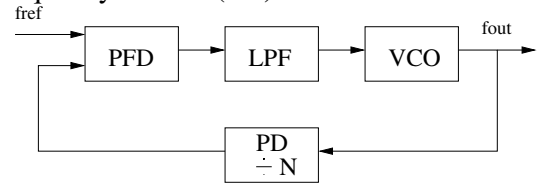

Fig. 1. Block diagram of a PLL.

A widely used approach for fast simulation of PLLs is to use phase macromodels to represent each component of a PLL and to simulate the system of macromodels. For example, Figure 2 shows a phasedomain model of a PLL in [14]. The PD is modeled as $K_{p d} f\left(\phi_{1}, \phi_{2}\right)$, where $\phi_{1}, \phi_{2}$ are the phases of the reference signal and the output of the VCO, respectively. $K_{p d}$ represents the gain of the PD and function $f($.$) describes the transformation relations between the phase differ-$ ence and output voltage. For instance, if a mixer is used as the PD, then

$$
f\left(\phi_{1}, \phi_{2}\right)=\sin \left(\phi_{1}-\phi_{2}\right)+\sin \left(\phi_{1}+\phi_{2}\right) .
$$

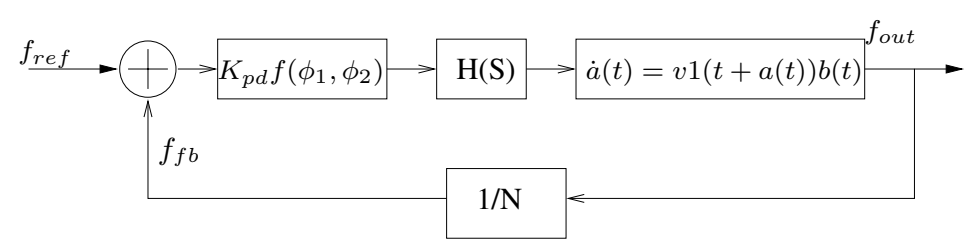

Fig. 2. Phase domain model of a PLL.

The LPF is often modelled by a system of linear ODEs as

$$
\begin{aligned}
\dot{x} & =A x+b(t), \\
y(t) & =d^{T} x(t) .
\end{aligned}
$$

where $b(t)$ is the output of the phase detector, $x(t)$ is a vector of circuit unknowns within the LPF and $y(t)$ is the output of the LPF ( $d$ is a vector which links the output to the rest of the system). The form of the ODE depends on the type of LPF used.

As mentioned in Section II-A, the VCO can be represented by the nonlinear macromodel (3) with the perturbation being the controlling voltage, i.e.,

$$
\frac{d \alpha(t)}{d t}=v_{v c o}^{T}(t+\alpha(t)) \cdot v_{c}(t)
$$

Thus the total phase at the output of the VCO is:

$$
\phi_{2}(t)=\omega_{0}(t+\alpha(t)) .
$$

Here $\omega_{0}$ is the free-running angular frequency of the VCO.

Combining all these phase domain macromodels together, we now have a system of ODE of much smaller size than that of the original PLL system:

$$
\left\{\begin{array}{l}
\dot{\alpha}(t)=v_{v c o}^{T}(t+\alpha(t))\left(d^{T} x(t)\right) \\
\dot{x}=A x+K_{p d} f\left(\phi_{1}, \phi_{2}\right)
\end{array}\right.
$$

where $\phi_{1}=\omega_{r} t$ ( $\omega_{r}$ is the frequency of the reference signal) and $\phi_{2}$ is define as (7).

We can solve the system (8) by transient simulation; as noted already, however, better alternatives can be desirable. For example, if the LPF has a low cutoff frequency, the PLL can take an extremely long time to lock $[15,16]$. For such situations, steady state methods (such as HB and shooting) are typically preferred, since they can "directly" find the locked state.

\section{HB SIMULATION ON PHASE MACROMODELS}

As mentioned before, phase deviations in oscillators can grow unboundedly when perturbed by external signals. Thus, they are not periodic waveforms; and as such, cannot be treated using normal HB. In this section, we first develop a generalization of $\mathrm{HB}$ for nonlinear phase macromodels (3) when oscillators are in lock. We then extend our approach to analyzing oscillators perturbed by DC signals. Finally, we demonstrate the use of our technique for PLL steady state simulations. 


\section{A. Oscillators in lock}

We assume that the unperturbed oscillator's frequency is $f_{0}$ and rewrite its PPV in the form $v_{1}(t)=\chi\left(f_{0} t\right)$, where $\chi(\cdot)$ is 1-periodic. For the moment, we also assume that the injection signal is a sinusoidal waveform with a frequency of $f_{1}$. Then the phase equation can be written as

$$
\dot{\alpha}(t)=\chi\left(f_{0}(t+\alpha(t))\right) \cdot \sin \left(2 \pi f_{1} t\right)
$$

When an oscillator is in lock, it changes its frequency to match the frequency of the perturbation signal, i.e., the phase of the oscillator, which is $2 \pi f_{0}(t+\alpha(t))$, "catches up" with the phase of the external signal. Hence

$$
f_{0}(t+\alpha(t))=f_{1} t+p\left(f_{1} t\right),
$$

where $p(\cdot)$ is a 1-periodic function yet to be determined. $p(\cdot)$ represents small phase variations within each period. Then,

$$
\alpha(t)=\frac{1}{f_{0}}\left[\left(f_{1}-f_{0}\right) t+p\left(f_{1} t\right)\right],
$$

It can be seen that the phase variation $\alpha(t)$ consists of two parts: an unboundedly growing part $\left(f_{1}-f_{0}\right) t$ which is responsible for locking the oscillator's frequency to that of the external signal, and a small periodic AC part $p\left(f_{1} t\right)$, which represents small phase variations in each period.

Plugging in (11) into (9), we get

$$
\left.\frac{1}{f_{0}}\left[\Delta f+f_{1} p^{\prime}\left(f_{1} t\right)\right)\right]=\chi\left(f_{1} t+p\left(f_{1} t\right)\right) \cdot \sin \left(2 \pi f_{1} t\right),
$$

where $\Delta f=f_{1}-f_{0}$. We can rewrite (12) in terms of a scaled time $t_{s}=f_{1} t$ as

$$
\frac{1}{f_{0}}\left[\Delta f+f_{1} p^{\prime}\left(t_{s}\right)\right]=\chi\left(t_{s}+p\left(t_{s}\right)\right) \cdot \sin \left(2 \pi t_{s}\right) .
$$

Observe that both sides of the above equation are periodic, with period 1. This means that we can expand everything in Fourier series and apply harmonic balance. Let $p(\cdot)$ be expressed in its Fourier series

$$
p\left(t_{s}\right)=\sum_{k=-M}^{M} P_{k} e^{j k 2 \pi t_{s}}
$$

The key computational step is: given (some guess for) $\left\{P_{i}\right\}$ (i.e., given the Fourier coefficients of $p\left(t_{s}\right)$ ), find the Fourier coefficients of $\chi(\cdot)$. At this point, we face a problem: suppose we sample $t_{s}$ uniformly with $N$ points, then we can find $p\left(t_{s}\right)$ easily enough at the samples using IDFTs. But now we cannot use DFTs for finding the Fourier coefficients of $\chi(\cdot)$, because $\chi(\cdot)$ is not sampled uniformly.

The problem is easily solved by moving to a bivariate function $\hat{\chi}(\cdot, \cdot)$ :

$$
\hat{\chi}\left(t_{1}, t_{2}\right)=\chi\left(t_{1}+p\left(t_{2}\right)\right) .
$$

Observe that $\hat{\chi}(\cdot, \cdot)$ is $(1,1)$-biperiodic in $t_{1}$ and $t_{2}$. This means that we can do a 2D DFT very easily to obtain its two-tone Fourier series coefficients

$$
\hat{\chi}\left(t_{1}, t_{2}\right)=\sum_{k=-M_{1}}^{M_{1}} \sum_{l=-M_{2}}^{M_{2}} \Xi_{k, l} e^{j 2 \pi\left(k t_{1}+l t_{2}\right)} .
$$

Now, given $\Xi_{k, l}$, we can obtain the Fourier coefficients of $\chi\left(t_{s}+\right.$ $\left.p\left(t_{s}\right)\right)=\hat{\chi}\left(t_{s}, t_{s}\right)$. Let

$$
\begin{aligned}
\chi\left(t_{s}+p\left(t_{s}\right)\right) & =\sum_{i=-M}^{M} \breve{\chi}_{i} e^{j 2 \pi i t_{s}} \\
& =\hat{\chi}\left(t_{s}, t_{s}\right) \\
& =\sum_{k=-M_{1}}^{M_{1}} \sum_{l=-M_{2}}^{M_{2}} \Xi_{k, l} e^{j 2 \pi(k+l) t_{s}} .
\end{aligned}
$$

Hence

$$
\breve{\chi}_{i}=\sum_{k=-M_{1}}^{M_{1}} \Xi_{k, i-k} .
$$

These look like anti-diagonal lines on the coefficient plane of $(k, l)$.

\section{B. Oscillators perturbed by DC inputs}

We assume that when a DC input is injected to an oscillator, it causes a shift in frequency $\Delta f$. The new frequency is $f_{0}+\Delta f$. Then,

$$
f_{0} \alpha(t)=\Delta f t+p\left(\left(f_{0}+\Delta f\right) t\right) .
$$

However, unlike the case in Section III-A, $\Delta f$ here is not known.

Now, the object is to find $\Delta f$ and $p(t)$ numerically. Plugging in (19) into (3), we get

$$
\begin{aligned}
& \left.\frac{1}{f_{0}}\left[\Delta f+\left(f_{0}+\Delta f\right) p^{\prime}\left(\left(f_{0}+\Delta f\right) t\right)\right)\right] \\
= & \chi\left(\left(f_{0}+\Delta f\right) t+p\left(\left(f_{0}+\Delta f\right) t\right)\right) \cdot b_{\mathrm{DC}} .
\end{aligned}
$$

Both sides of the above equation are periodic, with period $f_{0}+\Delta f$. Thus, we can expand everything in Fourier series and apply HB. Suppose we expand everything in $N=2 M+1$ harmonic components, as usual. Then we have $N+1$ unknowns - the $N$ harmonic components of $p(\cdot)$, plus $\Delta f$. But we have only $N$ harmonic balance equations. This "paradox" is explained by (3) having no "time reference" when $b(t)$ is a DC signal; i.e., if we define, for any $\tau$,

$$
\beta(t)=\alpha(t-\tau)-\tau,
$$

then $\beta(t)$ also satisfies (3), since

$$
\dot{\alpha}(t-\tau)=\chi\left(f_{0}(t-\tau+\alpha(t-\tau))\right) \cdot b_{\mathrm{DC}}
$$

is simply the same as choosing a different value of $t$ in (3). Therefore, we need to set an extra condition fixing the time reference, such as $\alpha(0)=0$. This is the same thing as saying that $p(0)=0$, or that $\sum P_{k}=1$. More generally, this means that one of the Fourier coefficients $P_{i}$ can be arbitrarily chosen; motivated by the right-hand side of (22), we choose $P_{0}=0$. Now we have $N+1$ unknowns and $N+1$ equations and the system can be solved uniquely.

\section{HB simulation of locked PLLs}

In PLL simulation, we use the nonlinear phase macromodel (6) to represent the VCO. We assume that the frequency of the free-running VCO in a PLL is $f_{0}$. If the PLL locks to a reference signal that has the same frequency as $f_{0}$, then the phase variation of VCO at PLL's steady state is purely periodic. More specificly, the phase of the VCO $\phi(t)$ has the form:

$$
\begin{aligned}
\phi(t)=f_{0}(t+\alpha(t)) & =f_{r} t+p\left(f_{r} t\right)+\theta \\
& =f_{0} t+p\left(f_{0} t\right)+\theta
\end{aligned}
$$


Here, $f_{r}$ is the reference frequency. $p($.$) is a periodic function which$ has the same frequency as the reference signal. It represents the effect of the high frequency component in the controlling voltage of the VCO, which is first generated by the phase detecter and then passes through the LPF (practical LPFs cannot perfectly filter out all high frequency components). $\theta$ is a constant phase offset when the PLL is in lock. Therefore,

$$
\alpha(t)=\frac{1}{f_{0}}\left(p\left(f_{0} t\right)+\theta\right)
$$

We can move the constant $\theta$ into $p($.$) since it only changes the DC$ component of $p($.$) . Denoting p_{1}\left(f_{0} t\right)=p\left(f_{0} t\right)+\theta$, we then have

$$
\alpha(t)=\frac{1}{f_{0}} p_{1}\left(f_{0} t\right) .
$$

Since $\alpha(t)$ is periodic, we can directly apply HB to the PLL phasedomain model (8).

However, when the PLL locks to a reference signal whose frequency is different from $f_{0}$, the $\mathrm{VCO}$ will change its frequency to match the reference frequency. Then the phase variation $\alpha(t)$ of $\mathrm{VCO}$ grows with time to "catch up" with the reference phase. In this case, $\alpha(t)$ is no longer periodic and hence the DAE system of the PLL phase-domain model (8) cannot be solved by HB.

In this case, the controlling voltage of the VCO consists of two components: a DC component responsible for frequency changes in the $\mathrm{VCO}$, and a small AC component representing the high frequency component leaked through the LPF. The frequency of the AC component is $f_{r}$. Now we can apply the PPV-HB technique developed in previous sections to modify the phase macromodel of the VCO so that we can apply HB on (8). More specifically, when the PLL locked to $f_{r}$, the phase of the VCO is:

$$
f_{0}(t+\alpha(t))=f_{r} t+p\left(f_{r} t\right)+\theta
$$

As before, we merge the constant $\theta$ into $p($.$) and denote the combined$ function as $p_{1}($.$) , i.e.,$

$$
\alpha(t)=\frac{1}{f_{0}}\left[\left(f_{r}-f_{0}\right) t+p_{1}\left(f_{r} t\right)\right]
$$

Plugging (27) into (8), we have

$$
\left\{\begin{array}{l}
\frac{1}{f_{0}} \dot{p_{1}}(t)+\frac{\Delta f}{f_{0}}=v_{v c o}^{T}\left(f_{r} t+p_{1}\left(f_{r} t\right)\right)\left(d^{T} x(t)\right), \\
\dot{x}=A x+K_{p d} f\left(\phi_{1}, \phi_{2}\right),
\end{array}\right.
$$

where $\Delta f=f_{r}-f_{0}, \phi_{1}=2 \pi f_{r} t$ and $\phi_{2}=2 \pi\left(f_{r} t+p_{1}\left(f_{r} t\right)\right)$. Note that $x$ are the node voltages inside the LPF and are periodic with frequency $f_{r} . p_{1}($.$) is also periodic with the same frequency. Thus,$ we can apply HB on (28). The Fourier coefficients of $v_{v c o}^{T}($.$) can be$ evaluated using the technique developed in Section III-A.

\section{ApPLiCATION AND VAlidation}

In this section, we apply PPV-HB to LC and ring oscillators, as well as a PLL. Simulation results confirm good matches against SPICElevel transient analysis, with speedups of orders of magnitude obtained. All simulation were performed using a MATLAB-based circuit/system simulation environment on an $2.4 \mathrm{GHz}$, Athlon XP-based PC running Linux.

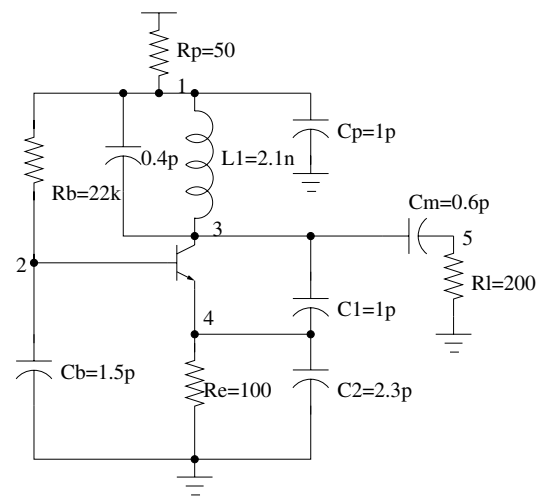

Fig. 3. A $4 \mathrm{GHz}$ Colpitts LC oscillator

\section{A. $4 \mathrm{GHz}$ Colpitts LC oscillator}

A Colpitts LC oscillator is shown in Figure 3. The circuit has a freerunning frequency $f_{0}$ of approximately $4 \mathrm{GHz}$. The circuit is perturbed by a sinusoidal voltage source in series with the inductor L1.

When the oscillator is locked to an external signal, the phase variation $\alpha(t)$ grows linearly with time, as shown in (11). However, it is difficult to predict whether the oscillator is in lock using transient simulation on the phase macromodel of the oscillator (3), although it is far more efficient and convenient than transient simulation on the oscillator itself. It is possible to reach the wrong conclusion by observing transient simulation results of (3) for a given number of cycles, especially when approaching the lock range of the injected signal ${ }^{1}$, since it is usually not known a priori how many cycles of transient simulation are needed to detect injection locking in oscillators.

For example, Figure 4 shows a transient simulation of (3) when the injected signal is $4 \times 10^{-3} \sin \left(2 \pi 1.01 f_{0} t\right)$. We need to simulate the phase macromodel for at least 200 cycles to see that the oscillator is actually not in lock. We can be easily misled by the fact that the phase variation $\alpha(t)$ grows linearly with time if we only perform transient simulation for fewer cycles, say 100 cycles.

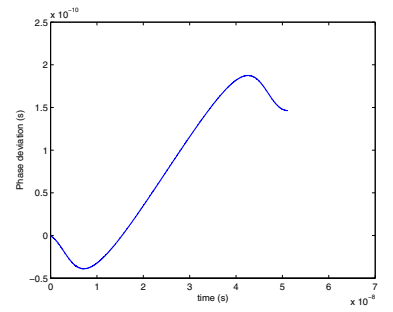

Fig. 4. Phase variation when the perturbation current is $4 \times$ $10^{-3} \sin \left(1.01 w_{0} t\right)$. The figure shows the simulation result for 200 cycles.

We now change the injection signal to be $4 \times 10^{-3} \sin \left(2 \pi 1.005 f_{0} t\right)$. Figure 5 shows the transient simulation result for 200 cycles. It seems that the oscillator is in lock. We then use our PPV-HB technique to solve the steady state of the locked oscillator. It only takes 4 iterations for $\mathrm{HB}$ to converge with a reasonable initial guess. It confirms that the oscillator is in lock. Figure 6(a) shows the steady state of $p(t)$ in (13). The phase variation can be recovered using (11), which is compared with the transient simulation result in Figure 6(b). A speedup of about 226 is obtained over the transient simulation on the phase macromodel (vs transient over 200 cycles).

\footnotetext{
${ }^{1}$ The lock range is the maximum frequency deviation from the oscillator's natural frequency.
} 


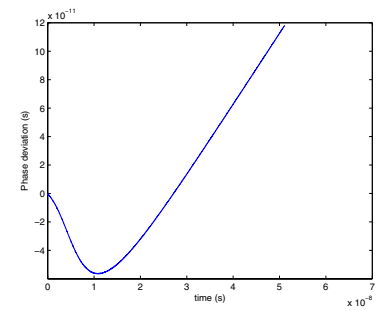

Fig. 5. Phase variation when the perturbation current is $4 \times$ $10^{-3} \sin \left(1.005 w_{0} t\right)$. The figure shows the simulation result for 200 cycles.

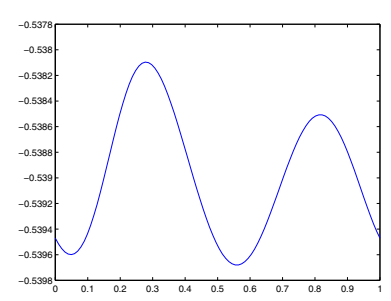

(a) Steady state of $p(t)$

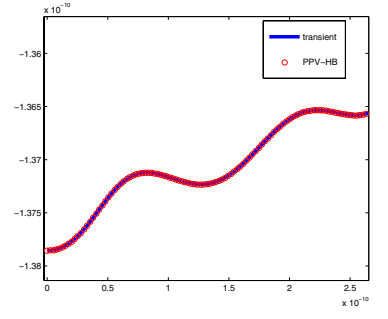

(b) Comparison of $\alpha(t)$
Fig. 6. Oscillator in lock: the perturbation is $4 \times 10^{-3} \sin \left(2 \pi 1.005 f_{0} t\right)$

When we use the perturbation $4 \times 10^{-3} \sin \left(2 \pi 1.01 f_{0} t\right)$, which cannot lock the oscillator as shown above, the PPV-HB does not converge. Therefore, our PPV-HB can be exploited as a useful approach to help predict injection locking: the oscillator is injection locked if PPV-HB converges. However, it is difficult to predict injection locking when PPV-HB does not converge, since convergence failure can result from many other causes. A modified approach is as follows: if the oscillator is in lock and PPV-HB converges, then slight changes to injection signal are unlikely to make large change to the steady state solution of PPV-HB. Then the solution from the previous PPV-HB (when oscillator is in lock) can be used as a good initial guess for the next PPV-HB (with injection frequency slightly changed). If, even after this simple continuation strategy, PPV-HB fails, our experience has been that it is highly likely that the oscillator is not locked ${ }^{2}$.

We also test our PPV-HB method on the case when the oscillator is perturbed by DC signals. Figure 7(a) shows the steady state of $p(t)$ when the perturbation strength is $7 \times 10^{-3} \mathrm{~V}$. The oscillator frequency solved is $0.99958 f_{0}$. The result is confirmed by transient simulation, as shown in Figure 7(b).

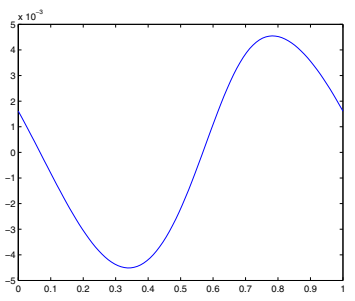

(a) Steady state of $p(t)$

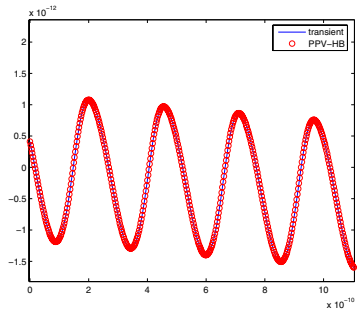

(b) Comparison of $\alpha(t)$
Fig. 7. Oscillator under DC perturbation: the perturbation is $7 \times 10^{-3}$

\footnotetext{
${ }^{2}$ We fully recognize that even though practically useful, this heuristic is rather unaesthetic; we are currently working on generalizations of PPV-HB that will solve this problem elegantly and robustly.
}

\section{B. 3 Stage Ring Oscillator}

A 3-stage ring oscillator with identical stages is shown in Figure 8. The free-running frequency of the oscillator is $1.53 \times 10^{5} \mathrm{~Hz}$. We inject a sinusoidal current into the output of the 2 nd stage.

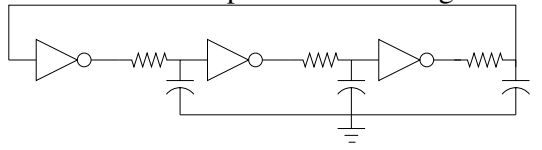

Fig. 8. A 3 stage oscillator with identical stages.

Figure 9(a) shows the steady state solution from our technique when the oscillator is in lock (the injection signal is $5 \times$ $\left.10^{-5} \sin \left(2 \pi 1.02 f_{0} t\right)\right)$. The phase variation recovered from the steady state solution is compared with transient simulation in Figure 9(b). They are in good agreement. We obtain a speedup of about 50 times in this example (we only run 100 cycles of transient simulation). We also test our technique when the injection frequency is $1.03 f_{0}$ and $1.04 f_{0}$ (the amplitude remains the same). HB simulations do not converge with good initial guesses (initial guesses are chosen using the approach outlined in Section IV-A) in both cases. The transient simulation confirms that oscillator is not locked in both cases. However, it takes many cycles for transient simulation to predict injection locking. For instance, it takes at least 300 cycles to see that the oscillator is not in lock when the injection frequency is $1.03 f_{0}$, as shown in Figure 10.

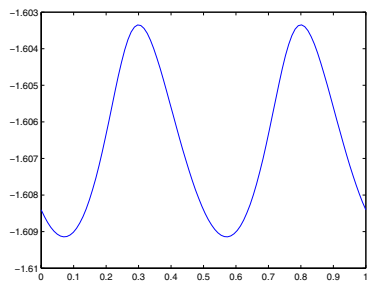

(a) Steady state of $p(t)$

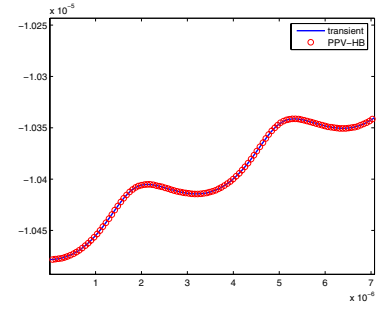

(b) Comparison of $\alpha(t)$
Fig. 9. Oscillator in lock: the perturbation is $5 \times 10^{-5} \sin \left(2 \pi 1.02 f_{0} t\right)$.

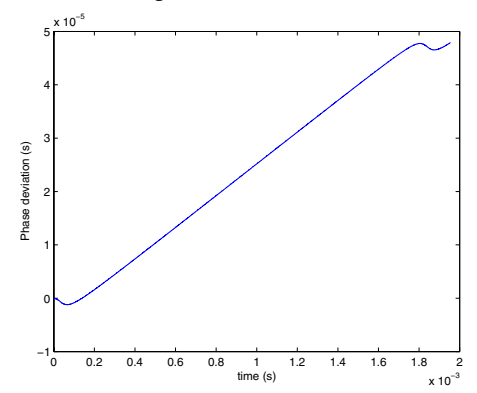

Fig. 10. Phase variation using transient simulation: the perturbation is $5 \times$ $10^{-5} \sin \left(2 \pi 1.03 f_{0} t\right)$ (the figure shows results for 300 cycles).

\section{PLL steady state simulation}

The PLL used is shown in Figure 11. In this PLL, a mixer is used as the phase detector (PD). The LPF has a high bandwidth; this allows high frequency components to pass through the LPF in significant quantity, exciting non-idealities in VCO behaviour. (Such effects are typically of great concern in today's integrated RFICs and serial data links.) The LC VCO has a free-running frequency $f_{0}$ of about $100 \mathrm{MHz}$; the original system has a size of 14 . We reduce it to a size of 4 using the phase-domain macromodels (8).

We first use a reference signal with a frequency of $f_{0}$. Figure 12(a) shows the phase variation $\alpha(t)$ of the VCO from transient simulation. 


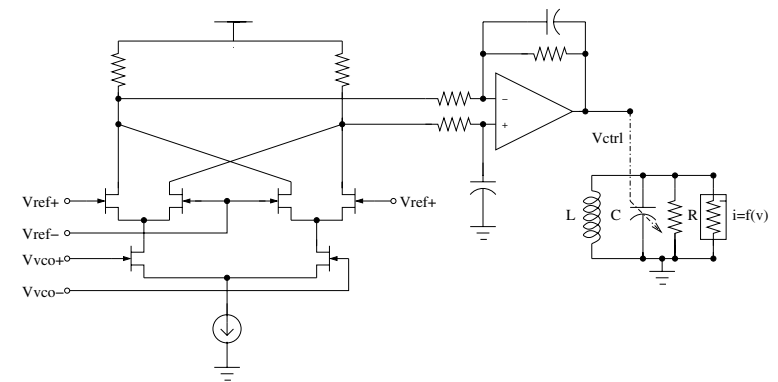

Fig. 11. Schematic of a PLL.

It confirms our previous conclusion that $\alpha(t)$ is purely periodic in this case. Thus we can directly apply HB on (8). We compare the steady state solution of $\alpha(t)$ with the result from transient simulation in Figure 12(b). As can be seen, they match well. We use 101 harmonics in the HB simulation. It matches transient simulation with 400 points per cycle, as shown in Figure 12(b). In this example, it takes about 300 cycles for the PLL to lock, resulting in a speedup of about 100 .

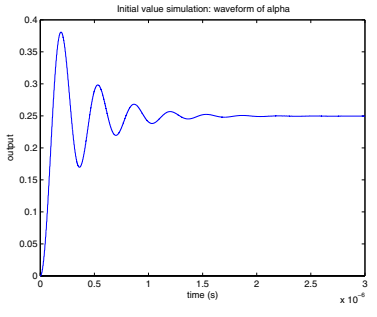

(a) $\alpha(t)$ from transient simulation.

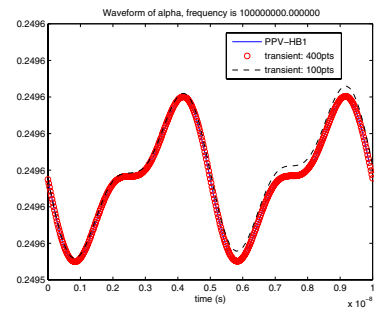

(b) Comparison of $\alpha(t)$.
Fig. 12. PLL locked to $f_{0}$.

We then lock the PLL to a reference signal of frequency $1.01 f_{0}$. The phase variation $\alpha(t)$ of the VCO (300 cycles) from transient simulation is shown in Figure 13(b). It can be seen that $\alpha(t)$ is not periodic, so HB does not apply. We use our PPV-HB technique to solve for the steady state of the PLL. Figure 14 shows the comparison between our results with transient simulation results at the controlling voltage, as well as $\alpha(t)\left(\alpha(t)\right.$ in our technique is recovered from $\left.p_{1}(t)\right)$. A speedup of 73 is obtained. Furthermore, the high bandwidth LPF we have used leads to a relatively fast lock of the PLL. For a LPF with lower bandwidth, the PLL takes longer to lock, resulting in larger speedups.

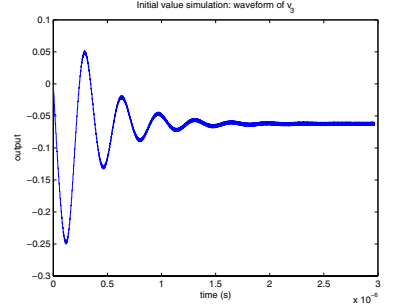

(a) The controlling voltage

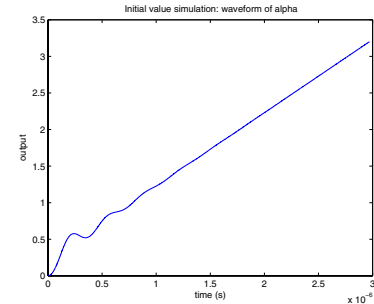

(b) $\alpha(t)$.
Fig. 13. Transient simulation result (300 cycles).

\section{CONClusions And Future Directions}

In the paper, we have presented a generalization and extension of HB to phase macromodels of oscillators. We have demonstrated the use of our technique to predict injection locking, as well as oscillator responses under DC perturbations. Our method, PPV-HB, enables the use of HB on system-level simulation of PLLs, resulting in improved

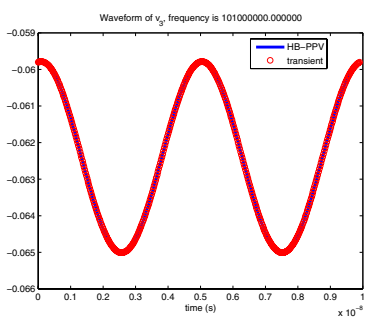

(a) The controlling voltage

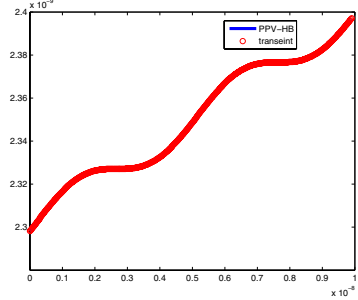

(b) $\alpha(t)$.
Fig. 14. Steady state of locked PLL (locked to $1.01 f_{0}$ ): comparison between PPV-HB results and transient simulation results.

efficiency and accuracy over transient simulation for several commonly used design tasks. We are currently extending PPV-HB to steady state simulation of unlocked oscillators, as well as fast transient envelope methods for phase macromodels of oscillators.

\section{REFERENCES}

[1] H. Rategh and T. Lee. Superharmonic injection locked frequency dividers. IEEE J. Solid-State Circuits, 34:813-821, June 1998

[2] S. Verma, H. Rategh, and T. Lee. A Unified Model for Injection-Locked Frequency Dividers. IEEE J. Solid-State Circuits, 38:1015-1027, June 2003.

[3] Prashant Goyal, Xiaolue Lai, and Jaijeet Roychowdhury. A fast methodology for first-time-correct design of plls using nonlinear phase-domain vco macromodels. In Proceedings of the 2006 conference on Asia South Pacific design automation, pages 291-296, 2006.

[4] P. Kinget, R. Melville, D. Long, and V. Gopinathan. An injection-locking scheme for precision quadrature generation. IEEE Journal of Solid-State Circuits, 37:845 - 851, 2002.

[5] F. Kärtner. Analysis of white and $f^{-\alpha}$ noise in oscillators. International Journal of Circuit Theory and Applications, 18:485-519, 1990.

[6] F. Kärtner. Determination of the correlation spectrum of oscillators with low noise. IEEE Transactions on Microwave Theory and Techniques, 37(1):90-101, 1989.

[7] A. Demir, A. Mehrotra, and J. Roychowdhury. Phase noise in oscillators: a unifying theory and numerical methods for characterization. IEEE Trans. Ckts. Syst. - I: Fund. Th. Appl., 47:655-674, May 2000.

[8] X. Lai and J. Roychowdhury. Capturing Oscillator Injection Locking via Nonlinear Phase-Domain Macromodels. IEEE Trans. Microwave Theory Tech., 52(9):2251-2261, Sep 2004.

[9] R. Adler. A study of locking phenomena in oscillators. Proc. Inst. Radio Eng., 34:351 - 357, Jun 1946.

[10] K. Kundert, J. White, and A. Sangiovanni-Vincentelli. Steady-State Methods for Simulating Analog and Microwave Circuits. Kluwer Academic Publishers, 1990.

[11] V. Rizzoli and A. Neri. State of the art and present trends in nonlinear microwave cad techniques. IEEE Trans. MTT, 36(2):343-365, Feb 1988.

[12] R.J. Gilmore and M.B. Steer. Nonlinear circuit analysis using the method of harmonic balance - a review of the art. part i. introductory concepts. Int. J. on Microwave and Millimeter Wave CAE, 1(1), 1991.

[13] A. Demir and J. Roychowdhury. A Reliable and Efficient Procedure for Oscillator PPV Computation, with Phase Noise Macromodelling Applications. IEEE Transactions on Computer-Aided Design of Integrated Circuits and Systems, pages 188-197, February 2003.

[14] Xiaolue Lai and J. Roychowdhury. Fast pll simulation using nonlinear vco macromodels for accurate prediction of jitter and cycle-slipping due to loop nonidealities and supply noise. In Proc. IEEE ASP-DAC, January 2005.

[15] Sergio Sancho and Almudena Suarez. Efficient analysis of phase-locked loops through a novel time- frequency approach, based on two envelope transient formulations. 2003 IEEE MTT-S International Microwave Symposium Digest, 3:2153-2156, June 2003.

[16] S. Sancho, A. Suarez, and J. Chuan. General envelope-transient formulation of phase-locked loops using three time scales. IEEE Transactions on Microwave Theory and Techniques, 52:1310-1320, April 2004. 